\title{
$\checkmark$ ENEI
}

Encontro Nacional de Economia Industrial e Inovação

\section{Uma análise empírica dos esforços inovativos como instrumento de Política Industrial Verde para países selecionados}

\author{
Rafael Moraes de Sousa (Doutorando em Economia pelo Programa de Pós-Graduação em \\ Economia da Universidade Federal de Uberlândia (PPGE-UFU); \\ Karina Palmieri de Almeida (Doutoranda em Economia pelo Programa de Pós-Graduação em \\ Economia da Universidade Federal de Uberlândia (PPGE-UFU);
}

\section{Resumo:}

Este trabalho examina a relação entre esforços inovativos verdes (públicos) e degradação ambiental de curto e longo prazo, mediante um painel de cointegração com 18 países selecionados para o período de 1995-2018. O Painel ARDL para cointegração foi especificado com variáveis representando efeitos de escala, composição e mudança tecnológica. Os resultados sugerem que níveis mais elevados de dispêndios públicos em recursos direcionados para o P\&D ambiental (esforços inovativos verdes), com intuito de gerar tecnologias verdes, possuem uma relação negativa com o crescimento dos impactos ambientais, i.e., tais investimentos têm uma boa expectativa de redução da degradação ambiental.

\section{Pallaviras-chave:}

Política Industrial Verde; Esforços inovativo; Degradação; Meio ambiente; Painel ARDL.

Código JEL: C23; O13; O25; Q58

Área Temática: 7: Tecnologias Sociais e Ambientais

7.3 Inovação, desenvolvimento e sustentabilidade 


\section{Introdução}

A humanidade está se aproximando de vários pontos de inflexão ecológicos, além dos quais mudanças ambientais abruptas e irreversíveis em grandes escalas geográficas provavelmente acontecerão (ROCKSTRÖM et al., 2009). Diante disso, verifica-se a necessidade de pensar novos sistemas tecnoinstitucionais necessários para separar o desenvolvimento econômico e o bem-estar humano do esgotamento de recursos e da produção de resíduos não degradáveis. Acredita-se que as iniciativas governamentais ainda têm sido tímidas no sentido de reduzir as emissões de gases de efeito estufa e minimizar os impactos que causam a degradação ambiental. Posto isso, verifica-se a necessidade de esforços conjuntos dos governos para estimular e facilitar o desenvolvimento de tecnologias verdes.

Embora a política industrial verde ainda não tenha assumido posição de destaque na agenda da maioria dos países, não obstante, o interesse no desenvolvimento de energias limpas e renováveis, bem como uma produção industrial fincada em tecnologias sustentáveis engendradas em uma pauta voltada às questões ambientais e mudanças climáticas têm se tornado uma preocupação constante. Tal preocupação decorre, sobretudo, de um cenário onde verifica-se pressões importantes sobre os recursos naturais do planeta que estão influenciando alguns componentes críticos do funcionamento básico do sistema terrestre.

O explosivo crescimento populacional, especialmente, a partir da Revolução Industrial, que intensificou-se no século XX, somado à expansão do sistema econômica global, passou a exigir quantidades crescentes de recursos naturais e gerar volumes cada vez maiores de emanações ao meio ambiente de rejeitos nocivos. Na década de 1970, a preocupação tornou-se mais intensa e consistia na possível escassez de recursos energéticos. Não obstante, hoje a preocupação reside, sobretudo, no que tangem os possíveis impactos de poluição global, em especial, nas mudanças climáticas, que vem originando o denominado "efeito estufa" (MÜLLER, 2007).

Nesse sentido, há um vínculo de possível debate entre investimento público direcionado às práticas de sustentabilidade, oriundos de uma discussão pautada por instrumentos de política industrial, com a preocupação da capacidade de resiliência ecossistêmica. Assim sendo, este artigo tem como objetivo principal investigar empiricamente como os estímulos públicos para o desenvolvimento de tecnologias verdes influenciam a degradação ambiental. Para cumprir esse objetivo, a estratégia empírica se dará por meio de uma Abordagem do Modelos Autorregressivo de Defasagens Distribuídas (ARDL) de Painel para cointegração. Para tanto, utilizou-se um banco de dados anual que abrange um grupo de 18 países para o período de 1995 a 2018.

O artigo foi construído a partir da hipótese de que o investimento público é um fator significativo para a promoção de tecnologias verdes, tendo em vista o contexto de ser um agente econômico especial. Além disso, pressupõe-se que países com esforços inovativos no âmbito ambiental possuem capacidade superior em reduzir os impactos ao meio ambiente.

Dessa forma, o artigo estrutura-se em três seções, além desta introdução e das considerações finais. Assim sendo, na sequência apresenta-se o referencial bibliográfico, que aborda brevemente o conceito de políticas industriais verdes, bem como seus benefícios e dificuldades. Posteriormente, apresenta-se, na seção 3, a metodologia, base de dados e o modelo empírico. Por fim, a seção 4 apresenta os resultados do presente trabalho.

\section{Política industrial Verde: Conceito, benefícios e dificuldades}

Embora verifique-se um crescente aumento do ativismo no que tange à sustentabilidade, utilização de energia renovável e investimento em tecnologia verde, existem algumas limitações quanto à implementação das políticas industriais verdes e sua capacidade de gerar resultados. Além das questões financeiras que tangem ao desenvolvimento tecnológico de cada país, um aspecto muito importante referese ao fato de que são ínfimos os incentivos para deixar de lado as velhas práticas de produção e adotar novos mecanismos mais sustentáveis que minimizem os impactos ambientais, além disso, muitas práticas são interpretadas como ameaças à competitividade da indústria nacional.

Nesse sentido, Rodrik (2014) argumenta que os benefícios da redução de gases poluentes na atmosfera representam um paradigma do bem público global, gerando fortes incentivos para que países individuais aproveitem os esforços de outros, em contrapartida, governos nacionais podem não estar tão interessados em tornar como interesses domésticos os interesses globais, demonstrando que as externalidades de P\&D do desenvolvimento de novas tecnologias verdes são, em muitos casos, globais, e não nacionais.

Embora muitas das tecnologias verdes já estejam disponíveis por meio das estratégias de 
desenvolvimento sustentável, os incentivos que orientam a alocação de recursos precisam mudar profundamente para interromper os atuais caminhos tecnológicos insustentáveis e mudar alguns subsistemas econômicos inteiramente trazendo benefícios para a sociedade global (IPCC, 2014). O engajamento recente de muitas nações às questões ambientalistas não significa necessariamente que estão genuinamente comprometidas com a causa. Muitos governos embarcaram pela oportunidade de criação de novos postos de trabalho, ou por razões de competitividade internacional, embates e pressões políticas.

Suzigan e Furtado (2006) definem política industrial como um mecanismo de coordenação de ações estratégicas do governo e de empresas visando o desenvolvimento de atividades indutoras de mudança tecnológica ou de solução de problemas identificados por esses atores no setor produtivo da economia. Nesse sentido, apesar da política ambiental ainda não assumir posição de destaque na agenda de alguns países, o interesse no desenvolvimento de energias limpas e renováveis, bem como tecnologias sustentáveis engendradas em uma pauta que dê o devido cuidado às questões ambientais e mudanças climáticas deveria estar entre os pilares que norteiam a política industrial no cenário internacional.

Destarte, existe um "trade off" irredutível entre crescimento econômico e preservação do meio ambiente. De modo geral, observa-se uma tendência dos governos em colocar o crescimento econômico acima dos objetivos ambientais, assim, tornam-se futuras as preocupações em reverter os danos ao meio ambiente. Por outro lado, existe uma vertente crítica por meio da qual argumenta-se que os ambientalistas tendem a colocar a preservação ambiental acima do crescimento e desenvolvimento econômico mundial.

Acerca disso, Daly (1999) afirma que a palavra crescimento é, por vezes, tratada como sinônimo de aumento de riqueza, pois muitos acreditam que devemos ter crescimento, pois somente se enriquecermos poderemos arcar com os custos da proteção ambiental e os problemas serão mais fáceis de serem resolvidos. Todavia, discute-se se o crescimento, na margem atual, está realmente nos tornando mais ricos, uma vez que, à medida que o crescimento nas dimensões físicas da economia humana e suas consequências vão além da escala ótima em relação à biosfera, na verdade nos torna mais pobres. Assim, o autor afirma que "o crescimento, que habitualmente chamamos de "crescimento econômico" enquanto estávamos abaixo da escala ideal, torna-se "crescimento antieconômico" uma vez que esse ótimo foi ultrapassado" (DALY, 1999, p. 5).

Sendo assim, Rodrik (2014) afirma que na prática, é improvável que obtenhamos uma política industrial puramente verde, com foco direto no desenvolvimento e na difusão de tecnologias verdes, em vez de competitividade, comércio, emprego ou motivos fiscais, uma vez que, objetivos indiretos, mas politicamente relevantes, como "empregos verdes", e demais motivos, provavelmente continuarão a apresentar uma plataforma mais atraente para a promoção da política industrial do que a energia alternativa ou tecnologias limpas e sustentáveis.

Mazzucato e Semieniuk (2016; 2018) advertem sobre a dificuldade de se financiar projetos de grande impacto com tecnologias verdes. Os custos de financiamento para esse tipo de projeto são bastante elevados frente às incertezas inerentes ao processo de inovação, pois os benefícios não são realizados no curto prazo, mas apenas depois de muitos anos, em que as condições econômicas, políticas e tecnológicas são bastante difíceis (senão impossíveis) de se prever (KEMP; NEVER, 2017).

Além disso, para Karp e Stevenson (2012) há um certo temor por parte da indústria em empreender $\mathrm{P} \& \mathrm{D}$ verde e/ou promover tecnologias verdes, isso pois não há certeza se a política futura tornará esses tipos de investimentos lucrativos. Os governos atuais não são capazes de assumir compromissos confiáveis sobre os tipos de políticas que estarão em vigor durante a vida desses investimentos.

Ainda nessa discussão, o risco atrelado ao investimento é um grande fator de aplicação ou não de determinados projetos. $\mathrm{O}$ setor privado busca investimentos em projetos de baixo risco, mas com alta intensidade de capital, pois, a despeito do elevado volume necessário de recursos, as incertezas quanto aos resultados do projeto são baixas. Por outro lado, em projetos de alta intensidade de capital e alto risco, (e.g., projetos de investimento em energia renovável) o setor público é o principal financiador, pois o custo para financiamento privado torna-se muito elevado.

Segundo Ferraz, Além e Madeira (2013) a existência dos bancos de desenvolvimento justifica-se, portanto, devido à necessidade de determinados setores e projetos de investimento que requerem financiamento, mas que denotam incerteza quanto a seu sucesso futuro devido à alta complexidade e grande dispêndio, fazendo com que encontrem dificuldade de acesso ao crédito privado. Em sua grande maioria, esses segmentos correspondem àqueles projetos de inovação que geram externalidades positivas e, por essa razão, caracterizam-se por apresentar retornos sociais relevantes, acima dos retornos privado, e transcendem o interesse econômico, e.g., as áreas da saúde e projetos econômicos ambientalmente e socialmente responsáveis. Assim sendo, o sistema financeiro privado prefere direcionar seus financiamentos para investimentos cujos resultados esperados sejam menos incertos (FERRAZ; ALÉM; MADEIRA, 2013).

Deste modo, ressalta-se que ao longo dos diversos estágios do processo de desenvolvimento, os 
Bancos Públicos vêm exercendo um papel fundamental no crescimento e desenvolvimento dos países, bem como no apoio aos projetos de inovação, atuando no financiamento da expansão da capacidade produtiva, visando atender aos segmentos do mercado para os quais o sistema financeiro privado não oferece instrumentos adequados de financiamento de longo prazo (FERRAZ; ALÉM; MADEIRA, 2013).

Assim, Kemp e Never (2017) apontam que em termos de operacionalização, a ecoinovação depende de políticas governamentais para lidar com falhas de mercado e problemas de falha sistêmica, além de problemas de economia política e relacionados às tomadas de decisões que são frequentemente fragmentadas. Não obstante, vale a pena ressaltar que o retorno do setor privado frente às tecnologias verdes está expressivamente abaixo do retorno social.

É diante dessa dificuldade que o setor público pode atuar, i.e., os governos podem usar a política industrial verde e seus instrumentos para promover esses investimentos tidos como arriscados. O setor público tem a possibilidade de adotar política industrial para promover o desenvolvimento de novas indústrias e a criação e adoção de novas tecnologias, uma vez que políticas públicas que aumentam a demanda por tecnologias verdes não apenas reduzem a poluição e o uso de recursos naturais, mas também promovem a inovação de tecnologias verdes e, portanto, o crescimento. Os tipos de política industrial incluem: créditos tributários; subsídios de insumos e produtos; investimento em inovação (P\&D); requisitos mínimos de uso; padrões e restrições comerciais (KARP; STEVENSON, 2012).

Sob essa perspectiva, Altenburg e Assmann (2017) afirmam que os governos podem promover a inovação em tecnologias verdes de várias maneiras. A primeira refere-se ao fato de que as empresas devem ser recompensadas por investir em P\&D, i.e., proteger suas invenções com patentes eficazes, mitigando assim o problema de falha de mercado. Uma vez que a capacidade de absorção tecnológica é vista por alguns autores como um determinante da capacidade de inovação de um país, a segunda maneira aponta que os governos devem investir em educação, treinamento tecnológico e infraestrutura de disseminação de conhecimento, incluindo acesso à internet. Em economias emergentes, esses fatores podem ajudar a fomentar a inovação em tecnologias verdes mais adequadas às condições locais. Do mesmo modo, em países menos desenvolvidos, isso pode facilitar a transferência de tecnologias verdes, mais complexas, em alguns setores, como o setor de energia eólica e solar (ALTENBURG; ASSMANN, 2017).

Nesse sentido, Rodrik (2014) destaca três elementos importantes que tem implicações específicas para o desenho institucional das políticas industriais verdes. O primeiro deles é denominado pelo autor de imersão. Para Rodrik (2014), uma estrutura de política industrial apropriada requer uma quantidade significativa de interação e comunicação entre os setores público e privado por meio de uma colaboração estratégica e coordenação com o objetivo de saber onde estão os gargalos mais significativos e como melhor aproveitar as oportunidades que essa interação revela reduzindo as incertezas.

O segundo elemento refere-se à disciplina, neste ponto o autor afirma que as empresas e indústrias que recebem ajuda do governo devem saber que não podem burlar o sistema e que o baixo desempenho resultará na remoção da assistência, portanto, são necessários mecanismos para disciplinar este sistema. Por fim, o terceiro elemento fundamental apontado pelo autor é a responsabilidade. Haja vista que o objetivo da política industrial verde é promover o bem público em geral, não os interesses das duas partes nessa relação, burocratas e empresas privadas, os órgãos públicos devem ter responsabilidade púbica e prestar contas tanto sobre seus sucessos quanto seus fracassos.

Diante disso, é importante ressaltar que a discussão sobre políticas industriais verdes também é influenciada pelos diferentes níveis de inovação tecnológica, desenvolvimento em P\&D e esforços inovativos de uma política industrial verde, uma vez que países desenvolvidos e países em desenvolvimento possuem dinâmicas distintas ao se tratar de crescimento econômico voltado para o uso de energias limpas e à uma produção industrial sustentável. Não obstante, verifica-se que a presença de indústrias verdes está concentrada em países de alta renda (DUTZ; SHARMA, 2012).

Logo, a presença e estímulos por parte do setor público traz consigo uma outra possibilidade, o demand pull. Podendo ser capaz de gerar políticas de inovação pelo lado da demanda, tais como: compras governamentais; exigências de conteúdo local associadas a requisitos de P,D\&I; normalização; regulação e suporte à demanda do setor privado (MACEDO, 2017).

Nesse sentido, ressalta-se que as funções exercidas pelo governo em sua representação do Estado alteram preços e a alocação de recursos nas economias de inúmeras formas, mesmo que sejam mínimas, i.e., toda aquisição pública e toda regulamentação influenciam a tomada de decisão dos agentes econômicos. A intervenção do Estado em um determinado mercado, afeta, direta ou indiretamente, preços, ociosidade, quantidades globais, rentabilidades e uma variada gama de outros indicadores financeiros empresariais. Portanto, a demanda por inovações pode ser maior ou menor em função da forma pela qual o Estado atua nos mercados (RAUEN, 2017).

Diante disso, o uso coordenado das políticas de inovação que atuam pelo lado da demanda por meio da influência do Estado nos mercados, pode ser evidenciado por um conjunto de ações que sinalizam 
as várias formas de participação do Estado na economia com o objetivo de estimular a demanda por produtos e/ou serviços inovadores por meio de compras públicas, regulação, políticas de clusters, dentre outros. Sendo assim, se as subvenções econômicas, e.g., atuam no sentido de garantir financiamento às empresas para que elas se desenvolvam e ofertem inovações e tecnologias, as compras públicas e os objetivos políticos explícitos e implícitos atuam sobre a formação e consolidação da demanda por inovações. Portanto, ao exercer uma função, mesmo que indireta e não associada à inovação, o Estado sinaliza suas preferências, podendo influenciar a criação de mercados para determinadas inovações (RAUEN, 2017).

\section{Metodologia e Base de Dados}

O presente trabalho utilizou-se dos dados de 18 países em um período de 24 anos (1995-2018), resultando em um total de 432 observações. A escolha dos países foi determinada por questões de disponibilidade de dados $^{1}$, sendo esse o grupo de países da OCDE com maior representatividade (tanto em regulariade, quanto em volume de recursos) de $\mathrm{P} \& \mathrm{D}$ ambiental. Assim, uma vez que os dados consistem em um painel de 18 países por 24 anos, onde $\mathrm{N}=18$, é menor que $\mathrm{T}=24$, o estimador GMM não é apropriado para esta análise. No entanto, quando T é maior do que N (como neste caso), a abordagem ARDL é apropriada e é, portanto, o método escolhido para a análise deste trabalho, sobretudo, dada a sua capacidade de manter estimadores robustos mesmo com amostras pequenas (ASTERIO E PILBEAM, 2020).

Tabela 1 - Países utilizados na amostra

\begin{tabular}{|c|c|c|c|c|}
\hline Região & Países & $\begin{array}{c}\text { Patentes em } \\
\text { Tecnologia ambiental } \\
\text { (Média 95-18) }\end{array}$ & $\begin{array}{c}\text { Ranking } \\
\text { Complexidade } \\
\text { Econômica }\end{array}$ & $\begin{array}{c}\text { Participação no PIB } \\
\text { mundial }\end{array}$ \\
\hline Europa & $\begin{array}{c}\text { Áustria } \\
\text { Alemanha } \\
\text { Bélgica } \\
\text { Dinamarca } \\
\text { Espanha } \\
\text { Grécia } \\
\text { Holanda } \\
\text { Irlanda } \\
\text { Itália } \\
\text { Noruega } \\
\text { Portugal } \\
\text { Suécia } \\
\text { Suíça } \\
\text { Reino Unido }\end{array}$ & $\begin{array}{l}0,53 \% \\
9,69 \% \\
0,42 \% \\
0,38 \% \\
0,62 \% \\
0,06 \% \\
1,02 \% \\
0,16 \% \\
1,33 \% \\
0,27 \% \\
0,05 \% \\
0,90 \% \\
0,90 \% \\
2,48 \% \\
\end{array}$ & $\begin{array}{c}6 \\
4 \\
21 \\
24 \\
32 \\
55 \\
27 \\
17 \\
14 \\
43 \\
33 \\
8 \\
2 \\
13\end{array}$ & $\begin{array}{l}0,40 \% \\
3,60 \% \\
0,48 \% \\
0,26 \% \\
1,51 \% \\
0,26 \% \\
0,79 \% \\
0,32 \% \\
2,06 \% \\
0,29 \% \\
0,28 \% \\
0,43 \% \\
0,46 \% \\
2,48 \%\end{array}$ \\
\hline Não Europa & $\begin{array}{c}\text { Estados Unidos } \\
\text { Canadá } \\
\text { Austrália } \\
\text { Japão } \\
\text { Mundo }\end{array}$ & $\begin{array}{c}27,80 \% \\
1,73 \% \\
0,46 \% \\
14,23 \% \\
\mathbf{1 0 0 , 0 0 \%}\end{array}$ & $\begin{array}{c}11 \\
39 \\
87 \\
1 \\
-\end{array}$ & $\begin{array}{c}15,76 \% \\
1,45 \% \\
0,98 \% \\
4,15 \% \\
\mathbf{1 0 0 , 0 0 \%}\end{array}$ \\
\hline
\end{tabular}

Fonte: Elaboração própria dos autores a partir de dados do OECD.STAT, WORLD BANK e ATLAS DA COMPLEXIDADE.

A Tabela 1 reúne os 18 países utilizados como amostra para o estudo, além disso apresenta algumas informações para justificar a relevância de nossa característica amostral. O primerio ponto de relevância está nos registros em patentes de tecnologias ambientais, sendo que os países do estudo representam, juntos, mais da metade de todas as patentes em tecnologias verdes do mundo $(63,03 \%)$, com destaque para os Estados Unidos que concentram mais de um quarto de todos os registros globais

\footnotetext{
${ }^{1}$ Referentes à alocação pública de recursos para P\&D ambiental.
} 
$(27,80 \%)$, na sequência Japão $(14,23 \%)$ seguido pela Alemanha $(9,69 \%)$ completam a tríade de nações fortes em registro de patentes para tecnologia ambiental.

Outro aspecto interessante diz respeito ao fato que 12 dos 18 países da amostra encontram-se entre as 30 economias mais complexas do mundo ${ }^{2}$. Hausmann e Hidalgo et al. (2009) desenvolveram uma metodologia para analisar a complexidade da estrutura produtiva de um país por meio de informações contidas em dados do comércio internacional, assim encontraram fortes indícios empíricos para sustentar o argumento de que uma economia é desenvolvida quando possui um grande êxito em reunir capacidades, habilidades e conhecimentos ( kills) produtivos, que por sua vez permitem que essa economia produza um grande número de bens de elevada complexidade. Mais do que isso, o número de capacidades existentes na cadeia de produção não determina apenas a complexidade produtiva atual do país, mas determina também o número e a complexidade dos bens que podem ser produzidos no futuro, haja vista que as capacidades futuras dependem das capacidades atuais (ALENCAR ET AL., 2018). Logo, a complexidade econômica é um bom indicador da capacidade de reunir skills orientados para o sistema produtivo, que, por sua vez, para os propósitos do presente estudo, também podem ser correlacionados com a capacidade de reunir conhecimento para desenvolver tecnologias verdes, i.e., é razoável presumir que economias mais complexas também são mais hábeis em desenvolver um sistema mais alinhado com o crescimento verde e, portanto, a nossa amostra está bem representada por economias complexas.

Dada a escolha do método e da amostra, o principal objetivo desta pesquisa é analisar como estímulos públicos para o desenvolvimento de tecnologias verdes influenciam a degradação ambiental, logo, a estratégia empírica se dará por meio de uma abordagem adotando Modelos ARDL de Painel Cointegrado. Para tanto, utilizou-se um banco de dados anual que abrange um grupo de 18 países, para o período de 1995 a 2018.

Para tanto, é preciso elencar as variáveis aplicadas no modelo (como controle) e na construção de um indicador (no qual consiste a variável de interesse do trabalho):

- A base de dados representativa dos Investimentos em P\&D:

Government budget allocations for R\&D. Apresenta dados sobre as dotações orçamentárias do Governo para P\&D (GBARD) por objetivo socioeconômico (SEO); (SEO): Meio Ambiente. Fonte: (OECD.STAT).

- A base de dados representativa do PIB Industrial:

Value added (current US\$). O valor adicionado é o produto líquido de um setor após somar todos os produtos e subtrair os insumos intermediários. Fonte: (World Bank).

- A base de dados para as variáveis de controle:

Escala. PIB per capita. US\$/PPP. Fonte: (World Bank). Sinal esperado: positivo, no sentido de que economias com rendas mais altas tendem a ter um padrão de consumo mais "agressivo" em relação à degradação ambiental.

Composição. Taxas ambientais em razão das demais taxas aplicadas. (\%). Fonte: (OECD.STAT). Sinal esperado: negativo, no sentido que taxas ambientais aplicadas podem auxiliar na mudança tecnológica para uma estrutura mais sustentável.

Portanto, a partir da definição dos dados é possível montar a variável de interesse do trabalho. Para tanto, o artigo adota como medida de esforço inovativo verde a construção, de forma adaptada, de um indicador de esforço tecnológico proposto por Zucoloto e Júnior (2005).

$$
E I V=\frac{\text { Dispêndios Públicos em P\&D ambiental }}{\text { PIB industrial (valor adicionado) }}
$$

Nesse sentido, a adaptação sugerida pelo trabalho, como variável representativa da inovação tecnológica verde, consiste em denotar uma medida de esforço tecnológico baseada na relação entre dispêndios em atividades de P\&D ambiental e o valor da produção industrial. Sucintamente, os países que despendem mais recursos em P\&D ambiental, como proporção do valor de seu PIB industrial, apresentam esforço tecnológico verde mais elevado. A variável escolhida tem, portanto, o intuito de revelar a importância da atividade de inovação ambiental ao correlacionar com dados de impacto ambiental.

O Gráfico 1 apresenta os resultados do indicador para os 18 países da amostra selecionada. Dividindo o período de análise em dois (1995-2007 e 2008-2018) nota-se que na maioria ampla dos países houve crescimento da participação do investimento público direcionado ao esforço de inovações

\footnotetext{
${ }^{2}$ Em uma base que contém 133 economias ranqueadas.
} 
ambientais, em proporção da produção industrial, o que pode sugerir um comportamento mais engajado do setor público com a pauta ambiental, sobretudo, por ser uma discussão amplamente debatida no cenário internacional, no qual há uma demanda crescente para que os países estejam alinhados com medidas de crescimento sustentável. Portanto, espera-se que o setor público seja um dos principais agentes a fomentar esse alinhamento com a pauta ambiental, especialmente, mediante política industrial verde e estímulos ao desenvolvimento de tecnologias verdes.

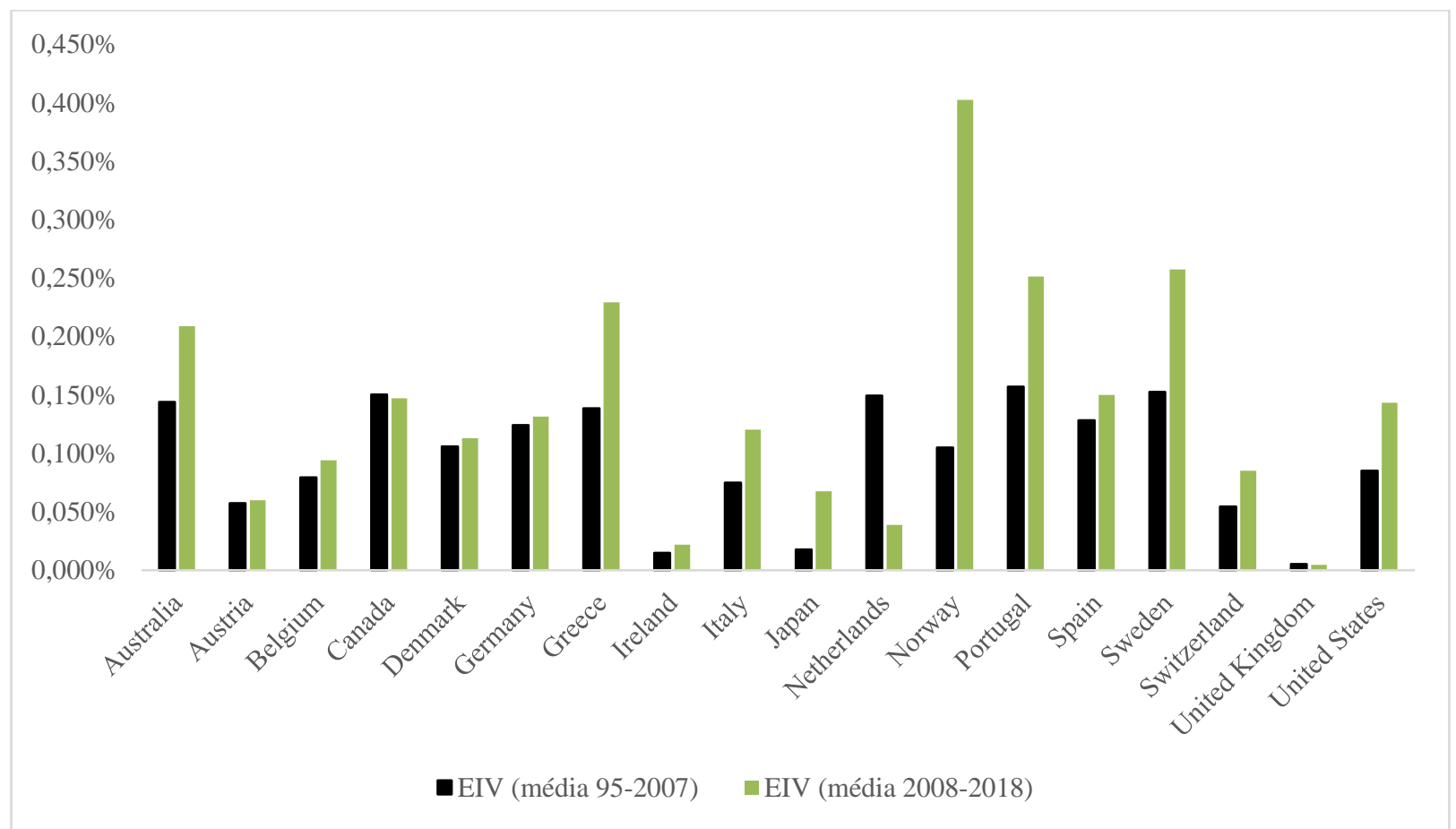

Gráfico 1 - Indicador de esforço inovativo verde para países selecionados

Fonte: Elaboração própria dos autores.

Nesse sentido, a construção lógica para a especificação de um modelo empírico parte da constatação da limitação dos recursos naturais disponíveis e da capacidade do meio ambiente de absorver os resíduos produzidos pela sociedade, uma vez que, há uma proeminente preocupação com uma possível insustentabilidade da produção material crescente e contínua (COLUSSO et al., 2013). Sob essa perspectiva, Mueller (2007) apregoa que se formou ao longo dos anos 1970 a ideia da existência de uma relação direta entre a degradação ambiental e o nível de produção. A possibilidade de "compatibilização" entre produção e proteção ambiental surge da ideia de desenvolvimento sustentável que, dentre as diversas definições encontradas na literatura, é mais usualmente conceituado como aquele capaz de satisfazer as necessidades da geração atual sem comprometer a capacidade das gerações futuras satisfazerem as suas próprias.

Carvalho e Almeida (2010) argumentam que aumentos na produção gerariam níveis de poluição (degradação) ambiental maiores, uma vez que, em um primeiro momento o país colocaria como prioridade o seu desenvolvimento e não o controle da qualidade da natureza. Posteriormente, quando este teria atingido determinando nível de crescimento, suas prioridades mudariam e com elas se observaria a redução da degradação ambiental. Essa transição denotaria a curva em forma de "U" invertido, denominada Curva de Kuznetz Ambiental (COLUSSO et al., 2013).

A CKA seria resultado de três efeitos, de acordo com Borghesi (2002, apud MUELLER, 2007): o efeito escala, o efeito composição e o efeito mudança tecnológica. $O$ efeito escala seria a consequência de que quanto maior a escala de produção de uma economia, maior é também a emissão de resíduos poluentes deste. O efeito composição faz referência à estrutura do processo produtivo, isto é, uma economia cujo setor de serviços ocupe a maior parcela do seu Produto Interno Bruto (PIB) poderia ser considerada uma economia mais "limpa". Mueller (2007) afirma ainda que o efeito composição agiria no sentido inverso ao efeito escala. E o efeito mudança tecnológica é a tendência a progredir para tecnologias mais eficientes e, de certa forma, mais "limpas". Segundo Mueller (2007), o efeito escala seria predominante em estágios iniciais de desenvolvimento e que posteriormente, com níveis maiores de desenvolvimento, se atribuiria pesos maiores aos efeitos composição e mudança tecnológica (COLUSSO et al., 2013). 
Em resumo, o modelo se baseia nesses três aspectos: o efeito escala (PIB per capita); o efeito composição (taxas ambientais); e a variável de interesse, o efeito mudança tecnológica (esforço inovativo verde). A próxima seção traz a estratégia empírica adotada no trabalho para estimar os efeitos acima citados.

\section{Modelo Empírico}

A metodologia proposta visa realizar um estudo empírico sobre os esforços inovativos verdes do setor público (indicador criado) para um grupo de países selecionados. Para tanto, será utilizada a metodologia de Modelos Autorregressivos de Defasagens Distribuídas (ARDL) para cointegração, como propostos por Pesaran e Shin (1999) e Pesaran et al. (2001). Não obstante, a estimativa de painel é escolhida neste estudo para controlar a heterogeneidade individual e para identificar características não observáveis (BALTAGI, 2005).

Além disso, Pesaran, Shin e Smith (1999) desenvolveram um modelo PMG (Pooled Mean Group), que é baseado em uma estrutura ARDL adaptada para um ambiente de dados em painel. De fato, os estimadores de probabilidade de PMG são usados para estimar coeficientes de longo prazo, capturando também o comportamento em grupo de restrições de homogeneidade e coeficientes de curto prazo, pela média do grupo utilizado, para obter, assim, parâmetros estimados de correção de erros e parâmetros de curto prazo (PESARAN; SHIN; SMITH, 1999).

A classificação dos dados no painel agrega as análises de dados em cross-section do grupo de indivíduos determinados durante um período de tempo. O uso de dados no painel tem algumas vantagens, tais como: o controle da heterogeneidade individual; os dados no painel também apresentam um número maior de dados informativos, mais variabilidade, menos colinearidade entre as variáveis, maior grau de liberdade e maior eficiência; uma análise de dados no painel é mais adequada aos estudos de ativação de ajuste; os dados no painel podem criar e testar modelos comportamentais mais complexos que dados crosssection ou dados em séries temporais, entre outras.

O modelo ARDL foi escolhido devido à sua vantagem sobre os testes de cointegração em variáveis não-estacionárias, como as desenvolvidas por Engle e Granger (1987), Phillips \& Hansen (1990) e Johansen (1991), bem como sobre modelos tradicionais de Vetores Autorregressivos (VAR). Além disso, os modelos ARDL tendem a ser mais eficientes para capturar relações de longo prazo em amostras pequenas de dados, e pode ser aplicado em um conjunto de variáveis com diferentes ordens de integração, sejam estacionárias I(0) ou não estacionárias I(1) (PESARAN; SHIN, 1999).

A abordagem ARDL consiste na verificação da existência de vetores de longo prazo entre um conjunto de variáveis. Confirmada esta relação, estimam-se os coeficientes de longo e curto prazos dos modelos, bem como a velocidade de ajustamento ao equilíbrio de longo prazo. Para tanto, o modelo PMGARDL é estimado na forma de vetores de correção de erros (ARDL-ECM), que pode ser especificado da seguinte maneira:

$\Delta(y)_{i t}=\phi_{i}(y)_{i, t-1} \beta_{i}^{\prime} x_{i t}+\sum_{j=1}^{p-1} \lambda_{i j}^{*} \Delta(y)_{i, t-j}+\sum_{j=0}^{q} \delta_{j=0}^{{*^{\prime}}^{\prime}} \Delta(x)_{i, t-j}+\mu_{i}+\varepsilon_{i t}$

Em que $\left(\Delta(y)_{i t}\right)$ é a variável dependente e $\Delta$ a primeira diferença, $\left(\alpha_{0}\right),(\mathrm{x})$ as variáveis independentes; $\phi_{i}=-\left(1-\sum_{j=1}^{p} \lambda_{i j}\right)$ representam o mecanismo de correção de erro para o i-nésmo grupo, $\beta_{i}=\sum_{j=0}^{q} \delta_{i j} \quad$ são os parâmetros de longo prazo para o i-nésmo grupo; $\lambda_{i j}^{*}=-\Sigma_{m=j+1}^{p} \lambda_{i m} j=1,2, \ldots, p-1$ são os parâmetros de curto prazo para o i-nésmo grupo; e $\left(\mathcal{E}_{t}\right)$ são os distúrbios do tipo ruído branco.

Para testar os efeitos dos objetivos propostos, pretende-se utilizar a abordagem ARDL e PMG de Pesaran et al. (2001), especificando 1 modelo que contém variáveis que serão observadas quanto aos efeitos de curto e longo prazo. Portanto, a equação especificada o modelo ARDL de painel deste trabalho é representada da seguinte maneira:

Variável dependente: Pegada Ecológica (footprint)

$\Delta(D A)_{i t}=\alpha+\alpha_{1} T+\beta_{1}(D A)_{i t-1}+\beta_{2}(E I V)_{i t-1}+\beta_{3}(C O M P)_{i t-1}++\sum_{j=}^{p} \beta_{4} \Delta(D A)_{i t-j}+$

$\sum_{j=}^{q} \beta_{5} \Delta(E I V)_{i t-j}++\sum_{j=}^{r} \beta_{6} \Delta(C O M P)_{i t-j}+\varepsilon_{t}$ 
Para fins deste trabalho, os modelos Painel ARDL (PMG) são aplicados na análise dos esforços inovativos verdes (\%) e mais duas variáveis de controle: PIB per capita (escala) e taxas ambientais (composição), além disso, denota-se $\alpha$ como uma constante e T como uma tendência de tempo.

\section{Resultados}

Primeiro, realizamos testes de raiz unitária do painel antes de realizar as principais estimativas; os testes são necessários para verificar se as variáveis são não estacionárias. Vários testes são realizados: Im et al. (2003) teste (IPS), Levin et al. (2002) teste (LLC) e teste IPS de segunda geração (CIPS) de Pesaran (2007). O teste LL é baseado na suposição de não heterogeneidade do parâmetro autorregressivo, enquanto o teste IPS permite a heterogeneidade enquanto a unidade CIPS mais segura relaxa a suposição de independência transversal da correlação contemporânea (EVIEWS USERGUIDE, 2020). Todos esses testes usam a hipótese nula de não estacionariedade. A seleção do comprimento de atraso é escolhida usando os critérios Bayesian-Schwarz. O objetivo dos testes é evitar que as séries tenham ordem de integração diferente de $\mathrm{I}(0)$ e I(1). A Tabela 2 apresenta os resultados para os testes, confirmando que as séries são aptas para a aplicação do método proposto.

Diante da metodologia empregada no trabalho, testes de cointegração de painel são fundamentais. O principal teste utlizado é chamado testes de cointegração de Pedroni (1996). De acordo com Eviews (2020), o teste de cointegração de Engle-Granger (1987) é baseado em um exame dos resíduos de uma regressão espúria realizada usando variáveis I (1). Se as variáveis forem cointegradas, os resíduos devem ser I (0). Por outro lado, se as variáveis não forem cointegradas, os resíduos serão I (1). Pedroni (1999) estende a estrutura Engle-Granger para testes envolvendo dados em painel.

Tabela 2 - Testes de Raiz Unitária

\begin{tabular}{c|c|c|c|c|c}
\hline & Levin-Lin-Chu & Im-Pesaran-Shin & ADF-Fisher & PP-Fisher & Decision \\
\hline $\begin{array}{c}\text { Esforço } \\
\text { Inovativo }\end{array}$ & -1.094 & -1.475 & 43.712 & 56.699 & Non \\
\hline \multirow{2}{*}{ Escala } & {$[0.137]$} & {$[0.701]$} & {$[0.176]$} & {$[0.015]$} & Stationary \\
\hline \multirow{2}{*}{ Composição } & -5.664 & -1.189 & 43.605 & 82.972 & Non \\
& {$[0.000]$} & {$[0.117]$} & {$[0.179]$} & {$[0.000]$} & Stationary \\
\hline \multirow{2}{*}{ DA } & 1.227 & 2.686 & 24.342 & 25.136 & Non \\
& {$[0.890]$} & {$[0.996]$} & {$[0.930]$} & {$[0.912]$} & Stationary \\
\hline
\end{tabular}

Fonte: Elaboração própria dos autores a partir do Eviews 9.

Pedroni (1999) propõe vários testes de cointegração que permitem interceptos heterogêneos e coeficientes de tendência em seções transversais. Considere a seguinte regressão:

$$
y i t=\alpha i t+\beta 1 i x 1 i, t+\beta 2 i x 2 i, t+\ldots+\beta M i x M i, t+\varepsilon i, t
$$

onde: i) y, x são I (1) variáveis, por suposição; ii) T é o número de observações ao longo do tempo $(\mathrm{t}=1,2, \ldots, \mathrm{T})$; iii) $\mathrm{N}$ é o número de indivíduos que fazem parte do painel $(\mathrm{i}=1,2, \ldots, \mathrm{N}) ; \mathrm{iv}) \mathrm{M}$ é o número de variáveis $(\mathrm{m}=1,2, \ldots, \mathrm{M})$; v) $\alpha$ i refere-se a efeitos individuais, que podem ser zero; vi) os parâmetros $\beta 1 \mathrm{i}, \beta 2 \mathrm{i}, \ldots, \beta \mathrm{Mi}$ podem variar entre membros individuais do painel, permitindo interceptos heterogêneos e coeficientes de tendência em seções transversais. Uma vez que a Equação (6) é estimada, os resíduos estimados são testados para não estacionariedade I (1), estimando a seguinte regressão auxiliar para cada seção transversal.

$$
\varepsilon_{i, t}=\rho_{i t} \varepsilon_{i, t-1}+\sum_{k=1}^{k_{i}} \rho_{i, k} \Delta(\varepsilon)_{i, t-k}+\mu_{i, t}
$$

Pedroni (1999) descreve alguns métodos para construir estatísticas apropriadas para testar a hipótese nula de não cointegração $\mathrm{\rho i}$ da equação dos resíduos. A Tabela 3 relata os resultados das estatísticas dentro (Within) e entre (Between) para o teste de dimensão. A cointegração é encontrada em pelo menos uma das estatísticas para o modelo estimado. Portanto, a evidência sugere uma relação de 
equilíbrio de longo prazo entre a variável de degradação ambiental e todas as outras variáveis, tanto as de interesse quanto as de controle.

Tabela 3 - Teste de Cointegração de Pedroni

\begin{tabular}{c|c|c|c|c|c|c|c}
\hline \multicolumn{7}{c}{ Within-dimension } \\
\cline { 2 - 8 } & \multicolumn{2}{c|}{ Modelo 1 } & \multicolumn{2}{c}{ Modelo 2 } & \multicolumn{2}{c}{ Modelo 3 } \\
\hline & Statistic & Prob. & Statistic & Prob. & Statistic & Prob. \\
\hline Panel v-Statistic & $-0,290061$ & 0.6141 & $-1,18977$ & 0.8829 & $-0,88024$ & 0.8106 \\
Panel rho-Statistic & $-9,838664$ & 0.0000 & $-5,57196$ & 0.0000 & $-2,80986$ & 0.0025 \\
Panel PP-Statistic & $-1,260833$ & 0.0000 & $-1,21987$ & 0.0000 & $-1,17249$ & 0.0000 \\
Panel ADF-Statistic & $-9,758524$ & 0.0000 & $-8,26017$ & 0.0000 & $-7,76121$ & 0.0000 \\
\hline \multirow{7}{*}{ Between-dimension } & & & \\
\hline & \multicolumn{2}{c}{ Modelo 1 } & Modelo 2 & Modelo 3 \\
\hline Statistic & \multicolumn{2}{c|}{ Prob. } & Statistic & Prob. & Statistic & Prob. \\
\cline { 2 - 8 } Group rho-Statistic & $-7,964722$ & 0.0000 & $-4,7084$ & 0.0000 & $-2,06977$ & 0.0192 \\
Group PP-Statistic & $-1,457744$ & 0.0000 & $-1,52112$ & 0.0000 & $-1,54428$ & 0.0000 \\
Group ADF-Statistic & $-1,092252$ & 0.0000 & $-8,95681$ & 0.0000 & $-8,83614$ & 0.0000 \\
\hline
\end{tabular}

Fonte: Elaboração própria dos autores a partir do Eviews 9.

H0: Note: p-values in brackets. Within-dimension with weighted statistic. Null: No cointegration.

Ademais, o Painel Autoregressivo com Defasagens Distribuidas (ARDL) é um método eficiente e superior a outros métodos de cointegração, permitindo que os regressores subjacentes exibam ordens de integração I (0) e I (1) ou uma mistura de ambos (PESARAN; SHIN, 1998). Além disso, uma vantagem específica para o caso deste trabalho, justificando sua aplicação, decorre do fato que o PMG permite a estimação de dados de painel macro com um intervalo de tempo de mais de 20 anos (ASTERIO E PILBEAM, 2020).

O PMG restringe o equilíbrio de longo prazo para ser homogêneo entre os países, ao mesmo tempo que permite heterogeneidade para o relacionamento de curto prazo. A relação de curto prazo centra-se na heterogeneidade específica do país, que pode ser causada por diferentes respostas de políticas de estabilização, choques externos ou crises financeiras para cada país.

Ainda sobre a escolha do PMG, uma outra vantagem de usar o método é que para uma seção transversal relativamente pequena de dados (18 países), o PMG é menos sensível à existência de outliers (PESARAN et al. 1999). Além disso, o problema de autocorrelação serial pode ser corrigido simultaneamente. O benefício de usar ARDL de painel com defasagens suficientes é a redução do problema de endogeneidade (PESARAN e SMITH, 1999), no qual é uma forte preocupação de pesquisadores ao adotarem a metodologia de dados em painel.

Paro o presente trabalho, os dados são provenientes de países de renda elevada, os quais apresentam comportamento semelhante no longo prazo, no que diz respeito ao crescimento econômico. Dito isto, visto que o estimador PMG pode aliviar o problema de endogeneidade com a inclusão de defasagens suficientes de todas as variáveis (Pesaran et al. 1999), a Tabela 4 apresenta os coeficientes de longo prazo para o modelo especificado, bem como o mecanismo de correção de erro. 
Tabela 4 - Coeficientes de Longo prazo e Mecanismos de Correção de erro (ECM)

Coeficientes de Longo prazo e ECM - Var. Dependente (Pegada ecológica)

\begin{tabular}{|c|c|c|c|c|c|c|}
\hline & \multicolumn{2}{|c|}{ Modelo 1} & \multicolumn{2}{|c|}{ Modelo 2} & \multicolumn{2}{|c|}{ Modelo 3} \\
\hline & Statistic & Prob. & Statistic & Prob. & Statistic & Prob. \\
\hline \multirow{4}{*}{$\begin{array}{c}\text { Esforço Inovativo } \\
\text { Escala } \\
\text { Composição } \\
\text { ARDL Lags }\end{array}$} & \multirow[t]{3}{*}{$-4.43 \mathrm{E}+16$} & \multirow[t]{3}{*}{0.0000} & \multirow{3}{*}{$\begin{array}{c}-4.67 \mathrm{E}+16 \\
1.72 \mathrm{E}+09\end{array}$} & \multirow{3}{*}{$\begin{array}{l}0.0000 \\
0.1463\end{array}$} & $-2.41 E+16$ & 0.0154 \\
\hline & & & & & $5.89 \mathrm{E}+09$ & 0.0606 \\
\hline & & & & & $-9.58 \mathrm{E}+12$ & 0.7161 \\
\hline & \multicolumn{2}{|c|}{$[1,1]$} & \multicolumn{2}{|c|}{$[1,1,1]$} & \multicolumn{2}{|c|}{$[1,2,2,2]$} \\
\hline Max. Lags & \multicolumn{2}{|c|}{2} & \multicolumn{2}{|c|}{3} & \multicolumn{2}{|c|}{3} \\
\hline ECM & -0.865390 & 0.0000 & -0.980582 & 0.0000 & -0.909918 & 0.0000 \\
\hline
\end{tabular}

Fonte: Elaboração dos autores a partir do Eviews 9.

A Tabela 4 reproduz os coeficientes de longo prazo do PMG quando a Pegada Ecológica (Footprint) é usada como variável dependente. Os resultados relativos aos Esforços Inovativos Verdes, variável de interesse, indicam que os coeficientes estimados são estatisticamente significativos em todas as estimativas. Portanto, as estimativas seguem alinhadas com o sinal esperado, de modo que os coeficientes negativos são encontrados nos Modelos 1, 2 e 3, sugerindo que níveis mais elevados (menores) de dispêndios públicos em recursos direcionados para o $\mathrm{P} \& \mathrm{D}$ ambiental, como proporção do valor de seu PIB industrial reduzem (aumentem) a degradação ambiental preservando, sobretudo, a resiliência ecossistêmica.

O coeficiente negativo esperado é um importante indicativo de que níveis mais altos (mais baixos) de investimentos em geração de novas tecnologias verdes estão associados à níveis mais baixos (mais altos) de impacto ambiental, o que corrobora com a perspectiva de que crescimento sustentável de longo prazo é indissociável de práticas produtivas capazes de mitigar a degradação ambiental, ou no melhor dos cenários estimular, concomitantemente, a resiliência do ecossistema e dos recursos naturais.

Para as demais variáveis, utlizadas como controle do modelo, os coeficientes para a escala (PIB per capita) são positivos em todos os modelos estimados e estatisticamente significativo no modelo 3 , enquanto a variável de composição (taxas ambientais) obteve o sinal negativo esperado, no entanto não se mostrou significativa. Isso indica que níveis mais altos (mais baixos) de renda per capita, estão diretamente associados a níveis mais altos (mais baixos) de degradação ambiental, um resultado esperado e amplamente já revelado pela literatura. Ademais, para a amostra utlizada as economias desenvolvidas, com menores níveis de renda, tendem a possuir forte impacto sobre a degradação ambiental, devido às suas características de consumo, mas, ao mesmo tempo, são os países com maiores capacidades tecnológicas para gerar inovações verdes, quando comparados aos países menos avançados, há, portanto, uma janela de investigação que carece de maiores buscas de evidências empíricas sobre qual dos dois fatores se sobrepõem.

Por fim, os ajustes de curto prazo relacionados à degradação ambiental, relatados na Tabela 4, revelam que todos os coeficientes do ECM são estatisticamente significativos com um sinal negativo (resultado esperado), confirmando um longo prazo de estável relação entre as variáveis especificadas. Quando a Pegada Ecológica é usada como variável dependente, o Mecanismo de Correção de Erros (ECM) varia de -0,86 a -0,98, com uma média de -0,9133. Isso significa que, em média, 91,33\% de uma perturbação de curto prazo, o que corresponde a um desvio do equilíbrio de longo prazo, é corrigido em um pouco mais de um ano, o que corresponde a um ajuste rápido dentro do modelo.

\section{Considerações Finais}

Este artigo teve como objetivo investigar o possível efeito dos esforços inovativos (do setor público) verdes sobre a degradação ambiental (Pegada Ecológica), para um painel de 18 economias avançadas. Antes de estimar os modelos empíricos, foi desenvolvido um indicador de esforço inovativo verde para calcular o dispêndio público em P\&D Ambiental de cada país da amostra. Além disso, foram utilizadas as seguintes variáveis de controle: PIB per capita representando os impactos de escala; e as taxas ambientais como representação de fatores da composição da produção. 
Para o período de 1995 a 2018, foi adotada uma abordagem de Painel ARDL para Cointegração (Pooled Mean Group), como metodologia econométrica. Os resultados encontrados indicam que níveis mais elevados de dispêndios públicos em recursos direcionados para o P\&D ambiental, com intuito de gerar tecnologias verdes, possuem uma relação negativa com o crescimento dos impactos ambientais, i.e., tais investimentos possuem uma boa expectativa de redução da degradação ambiental (se tomarmos como referência as características da estrutura produtiva vigente), preservando, sobretudo, a resiliência ecossistêmica, indo ao encontro com a perspectiva de que crescimento sustentável de longo prazo deve ser conduzido tendo em seu escopo de questões relevantes a mitigação da degradação ambiental e, concomitante, estímulo à resiliência dos ecossistemas e dos recursos naturais.

Além disso, os efeitos de curto prazo estimados mostram que em caso de choque de curto prazo os modelos estimados são corrigidos a uma taxa acelerada. Em outras palavras, os esforços inovativos verdes ajudam a direcionar o modelo para um ajuste estável de longo prazo. Por fim, também encontramos evidências de que outras variáveis de controle também são importantes. Esse foi o caso do PIB per capita, como proxy para o efeito de escala, conforme já advertido pela literatura, níveis mais altos de renda per capita, estão diretamente associados a níveis mais altos de degradação ambiental. 


\section{An empirical analysis of innovative efforts as an instrument of Green Industrial}

Policy for selected countries

Abstract: This paper examines the relationship between innovative green (public) efforts and short and long-term environmental degradation, through a cointegration panel with 18 countries selected for the period 1995-2018. The ARDL Panel for cointegration was specified with variable effects of scale, composition and technological change. The results obtained that higher levels of public expenditure on resources directed towards environmental R\&D (green innovative efforts), with the aim of green technologies, have a negative relationship with the growth of environmental impacts, i.e., that is. such investments have a good expectation of reducing environmental degradation.

Keywords: Green Industrial Policy; Innovative efforts; Degradation; Environment; Panel ARDL.

\section{Referências bibliográficas}

ALENCAR, JÚLIA F. L. et al. Complexidade econômica e desenvolvimento: Uma análise do caso latinoamericano. Novos estud. CEBRAP, São Paulo, v. 37, n. 2, p. 247-271, ago. 2018. Disponível em: http://www.scielo.br/scielo.php?script=sci_arttext\&pid=S010133002018000200247\&lng=en\&nrm=iso. Acesso em: 06 abr. 2021.

ALTENBURG, T.; ASSMANN, C. (Eds.). Green Industrial Policy: Concept, Policies, Country Experiences. Geneva, Bonn: UN Environment; German Development Institute / Deutsches Institut für Entwicklungspolitk (DIE). 2017.

ASTERIOU, D.; PILBEAM, K. \& PRATIWI, C.E. Public debt and economic growth: panel data evidence for Asian countries. J Econ Finan 45, 270-287. 2021.

BALTAGI, B. H. Econometric analysis of panel data. John Wiley \& Sons pp:4-8. 2005.

BALTAGI, B. H. The oxford handbook of panel data. Oxford University Press, USA. 2014.

CARVALHO, T. S; ALMEIDA, E. A hipótese da Curva de Kuznets Ambiental Global: uma perspectiva econométrico-espacial. Est. Econ., São Paulo, v. 40, n 3, p. 587-615, jul.-set. 2010.

COLUSSO, M. V.; SILVA \& PARRÉ, JOSÉ \& ALMEIDA, E. Degradação Ambiental e Crescimento Econômico: a Curva de Kuznets Ambiental para o Cerrado. Revista de Economia e Agronegócio. 10. 335. 10.25070/rea.v10i3.207. 2012.

DALY, H. E. Uneconomic growth: in theory, in fact, in history, and its relation to globalization. Clemens Lectures Series, Saint's John University, 1999.

DUTZ, M. A.; SHARMA, S. Green growth, technology and innovation. The World Bank, Policy Research Working Paper, 47 p. 2012.

ENGLE, R. F.; GRANGER, C. W. J. "Co-Integration and Error Correction: Representation, Estimation, and Testing." Econometrica, vol. 55, no. 2, 1987, pp. 251-276. JSTOR. Disponível em: www.jstor.org/stable/1913236. Accesso em: 21 dez. 2020.

FERRAZ, J. C.; ALÉM, A. C. D.; MADEIRA, R. F. A contribuição dos bancos de desenvolvimento para o financiamento de longo prazo. Revista do BNDES, Rio de Janeiro, n. 40, p. 5-42, dez. 2013. 
Hidalgo, César A.; Hausmann, Ricardo. "The Building Blocks of Economic Complexity". PNAS, v. 106, n. 26, pp.10570-10575, 2009.

IM, K. S.; PESARAN M. H., SHIN, Y. Testing for unit roots in heterogeneous panels. J Econ 115(1):53-74 Kumar, M. and Woo, J., 2010. Public debt and growth. IMF Working Papers, no. 10/174, pp.1-47. Washington: International Monetary Fund. 2003.

IPCC - Intergovernmental Panel on Climate Change: Climate Change 2014: Mitigation of Climate Change. Contribution of Working Group III to the Fifth Assessment Report of the Intergovernmental Panel on Climate Change [Edenhofer, O. et al. (eds.)]. Cambridge, United Kingdom and New York, NY: USA Cambridge University Press. 2014.

JOHANSEN, S. Estimation and Hypothesis Testing of Cointegration Vectors in Gaussian Vector Autoregressive Models. Econometrica, 59(6), p. 1551-1580. 1991.

KEMP, R.; NEVER, B. Green transition, industrial policy, and economic development, Oxford Review of Economic Policy, 33(1), 66-84. 2017.

KARP; STEVENSON. Green Industrial Policy: Trade and Theory. Policy Research Working Paper; No. 6238. World Bank, Washington, DC. 2012.

LEVIN A.; LIN C.; CHU C. J. Unit root tests in panel data: asymptotic and finite-sample properties. J Econ, 108(1):1-24. 2002.

MACEDO, M. M. Fundamentos das políticas de inovação pelo lado da demanda no Brasil. In: RAUEN, André Tortato (org.). Políticas de inovação pelo lado da demanda no Brasil. Brasília: Ipea, 2017. p. 481.

MAZZUCATO, M. E SEMIENIUK, G. Financing renewable energy: Who is financing what and why it matters. Technological Forecasting \& Social Change,127, 8-22. 2018.

MÜELLER, C. C. Os economistas e as relações entre o sistema econômico e o meio ambiente. Brasília: UnB, 2007.

PEDRONI, P. Fully modified OLS for heterogeneous cointegrated panels and the case of purchasing power parity. Manuscript, Department of Economics, Indiana University 5:1-45. 1996.

PEDRONI, P. Critical values for cointegration tests in heterogeneous panels with multiple regressors. Oxford Bulletin of Economics and Statistics, 61(1), p. 653-670. 1999.

PESARAN, M. H.; SHIN, Y.; SMITH, R. P. Pooled mean group estimation of dynamic heterogeneous panels. Journal of the American Statistical Association, 94(446), p. 621-634. 1999.

PESARAN, M. H.; SHIN, Y. An Autoregressive Distributed-Lag Modelling Approach to Cointegration Analysis. In: Strom, S. (ed.). Econometrics and Economic Theory in the 20th Century, p. 371413. 1999.

PESARAN, M. H.; SHIN, Y.; SMITH, R. J. Bounds Testing Approaches to the Analysis of Level Relationships. Journal of Applied Econometrics, 16, p. 289-326. 2001.

PHILLIPS, P. C. B.; HANSEN, B. E. Statistical Inference in Instrumental Variables Regression with I(1) Processes. Review of Economic Studies, 57, p. 99-125. 1990.

ROCKSTRÖM, J., STEFFEN, W., NOONE, K., PERSSON, A., CHAPIN, F.S., LAMBIN, E.R., LENTON, T.M., SCHEFFER, M., FOLKE, C., SHELLNHUBER, H.J., NYKVIST, B., WIT, C.A. de, HUGHES, T., VAN DER LEEUW, S., RODHE, H., SÖRLIN, S., SNYDER, P.K., COSTANZA, R., SVEDIN, U., FALKENMARK, M., KARLBERG, L. CORELL, R.W., FABRY, V.J., HANSEN, J., WALKER, B., LIVERMAN, D., RICHARDSON, K., CRUTZEN, P., FOLEY, J. A safe operating space for humanity. Nature 461, p. 472-475. 2009.

RODRIK, D. Green industrial policy, Oxford Review of Economic Policy, 30(3), 469-491. 2014. 
RAUEN, A. T. Racionalidade e primeiros resultados das políticas de inovação que atuam pelo lado da demanda no Brasil. In: RAUEN, A. T. (org.). Políticas de inovação pelo lado da demanda no Brasil. Brasília: Ipea, 2017. p. 481.

SUZIGAN, W.; FURTADO, J. Política Industrial e Desenvolvimento. Revista de Economia Política, vol. 26, n² 2 (102), pp. 163-185 abril-junho/2006.

ZUCOLOTO, G. F.; TONETO JUNIOR, R. Esforço tecnológico da indústria de transformação brasileira uma comparação com países selecionados. Revista de Economia Contemporânea, Rio de Janeiro, 9(2): 337-365, mai./ago. 2005. 
Anexos

Anexo 1 - Total de Patentes - Tecnologias ambientais (1995 - 2018)

\begin{tabular}{c|c|c|c|c|c|c|c}
\hline Região & Países & $\mathbf{1 9 9 5}$ & $\mathbf{1 9 9 6}$ & $\mathbf{1 9 9 7}$ & $\mathbf{1 9 9 8}$ & $\mathbf{1 9 9 9}$ & $\mathbf{2 0 0 0}$ \\
\hline \multirow{5}{*}{ Europa } & Áustria & $1.463,47$ & $1.684,87$ & $1.836,23$ & $2.008,51$ & $2.221,21$ & $2.386,07$ \\
& Bélgica & $1.337,83$ & $1.443,61$ & $1.676,37$ & $1.795,63$ & $1.989,94$ & $1.945,49$ \\
& Dinamarca & 990,62 & $1.062,82$ & $1.275,28$ & $1.551,88$ & $1.674,74$ & $1.914,83$ \\
& Alemanha & $35.391,84$ & $39.189,74$ & $42.772,61$ & $45.894,04$ & $48.961,08$ & $51.017,50$ \\
& Grécia & 68,78 & 86,48 & 98,83 & 119,29 & 117,15 & 116,00 \\
& Irlanda & 285,20 & 359,15 & 415,63 & 602,45 & 683,82 & 700,99 \\
& Itália & $4.920,27$ & $5.310,30$ & $5.862,42$ & $6.097,77$ & $6.367,08$ & $6.624,13$ \\
& Holanda & $3.261,12$ & $3.865,05$ & $4.164,68$ & $4.329,27$ & $4.763,03$ & $5.380,47$ \\
& Noruega & $1.192,95$ & $1.447,82$ & $1.425,45$ & $1.467,32$ & $1.562,55$ & $1.629,19$ \\
& Portugal & 43,33 & 65,78 & 59,33 & 96,42 & 109,02 & 124,00 \\
& Espanha & $1.535,78$ & $1.731,47$ & $1.965,02$ & $2.181,38$ & $2.577,96$ & $2.761,41$ \\
& Suécia & $2.505,95$ & $2.963,73$ & $3.420,38$ & $3.581,14$ & $3.831,00$ & $4.362,36$ \\
& Suíça & $3.232,22$ & $3.393,39$ & $3.687,22$ & $3.878,79$ & $4.134,64$ & $4.468,57$ \\
& Reino Unido & $7.174,93$ & $8.655,07$ & $9.653,01$ & $12.317,92$ & $13.791,53$ & $14.764,58$ \\
\hline & Estados Unidos & $96.600,49$ & $95.340,39$ & $109.832,04$ & $111.390,27$ & $119.835,78$ & $137.865,40$ \\
& Canadá & $5.209,58$ & $5.478,20$ & $6.673,45$ & $7.034,60$ & $7.655,20$ & $8.429,54$ \\
& Austrália & $1.200,03$ & $1.179,38$ & $1.240,86$ & $1.344,17$ & $1.472,12$ & $1.527,87$ \\
& Japão & $44.097,55$ & $48.673,33$ & $52.837,25$ & $54.086,03$ & $60.198,92$ & $71.248,29$ \\
& Mundo & 278.262 & 294.833 & 321.805 & 346.515 & 378.151 & 451.073 \\
\hline
\end{tabular}

(Continua...)

\begin{tabular}{c|c|c|c|c|c|c|c}
\multicolumn{1}{c}{ (Continuação) } \\
\hline $\mathbf{2 0 0 1}$ & $\mathbf{2 0 0 2}$ & $\mathbf{2 0 0 3}$ & $\mathbf{2 0 0 4}$ & $\mathbf{2 0 0 5}$ & $\mathbf{2 0 0 6}$ & $\mathbf{2 0 0 7}$ & $\mathbf{2 0 0 8}$ \\
\hline $2.626,94$ & $2.623,88$ & $2.754,94$ & $2.788,02$ & $2.870,50$ & $3.153,27$ & $3.153,73$ & $3.059,13$ \\
$1.908,42$ & $2.061,93$ & $2.180,19$ & $2.277,38$ & $2.489,21$ & $2.432,27$ & $2.496,22$ & $2.449,78$ \\
$1.966,02$ & $2.001,09$ & $2.168,49$ & $2.144,39$ & $2.266,47$ & $2.214,38$ & $2.512,20$ & $2.627,61$ \\
$50.388,23$ & $48.986,44$ & $49.791,67$ & $52.473,99$ & $52.889,24$ & $53.791,83$ & $55.379,64$ & $55.940,06$ \\
155,09 & 184,72 & 222,89 & 236,48 & 292,91 & 299,59 & 323,20 & 405,94 \\
835,62 & 811,01 & 762,26 & 806,20 & 865,42 & 913,55 & $1.007,95$ & $1.048,70$ \\
$5.902,25$ & $6.304,27$ & $6.781,05$ & $7.100,86$ & $7.702,60$ & $7.897,91$ & $7.863,37$ & $8.742,66$ \\
$5.598,21$ & $6.003,96$ & $6.012,18$ & $6.470,22$ & $6.438,78$ & $6.292,75$ & $6.140,37$ & $6.433,52$ \\
$1.507,25$ & $1.539,78$ & $1.380,27$ & $1.289,03$ & $1.548,47$ & $1.185,67$ & $1.181,91$ & $1.157,48$ \\
162,65 & 179,53 & 207,55 & 189,43 & 263,55 & 316,95 & 402,44 & 448,28 \\
$2.914,90$ & $3.076,02$ & $3.218,14$ & $3.577,69$ & $3.937,74$ & $3.862,27$ & $3.745,83$ & $3.727,54$ \\
$4.126,13$ & $3.970,40$ & $4.361,83$ & $4.731,22$ & $4.749,90$ & $5.202,43$ & $5.721,19$ & $6.047,34$ \\
$4.477,94$ & $4.436,79$ & $4.667,05$ & $4.767,09$ & $4.909,08$ & $4.881,24$ & $4.741,76$ & $5.013,14$ \\
$14.865,10$ & $14.159,60$ & $14.373,67$ & $13.985,96$ & $13.838,06$ & $14.051,28$ & $14.398,06$ & $14.094,16$ \\
\hline $150.927,59$ & $152.441,48$ & $153.659,04$ & $157.287,14$ & $161.364,54$ & $163.154,29$ & $163.677,75$ & $153.306,48$ \\
$9.116,43$ & $9.361,38$ & $9.494,17$ & $9.875,43$ & $10.416,54$ & $10.779,93$ & $10.765,20$ & $9.933,17$ \\
$1.577,03$ & $1.958,97$ & $2.063,44$ & $2.335,83$ & $3.039,87$ & $2.954,13$ & $2.952,39$ & $3.158,93$ \\
$71.310,13$ & $74.078,86$ & $77.944,93$ & $85.393,30$ & $87.058,66$ & $84.677,49$ & $85.577,00$ & $80.807,71$ \\
480.993 & 476.092 & 526.248 & 598.314 & 643.818 & 668.386 & 683.674 & 654.742 \\
\hline
\end{tabular}

(Continua...) 
Anexo 1 - Total de Patentes - Tecnologias ambientais (1995 - 2018)

\begin{tabular}{|c|c|c|c|c|c|c|c|c|c|}
\hline 2009 & 2010 & 2011 & 2012 & 2012 & 2014 & 2015 & 2016 & 2017 & 2018 \\
\hline $3.223,50$ & $3.364,62$ & $3.328,84$ & $3.549,49$ & $3.576,37$ & $3.646,74$ & $3.624,87$ & $3.651,09$ & $3.565,45$ & $3.430,01$ \\
\hline $2.391,18$ & $2.508,11$ & $2.685,93$ & $2.630,07$ & $2.496,03$ & $2.583,18$ & $2.767,66$ & $2.942,69$ & $2.873,41$ & $2.752,37$ \\
\hline $2.403,16$ & $2.482,45$ & $2.697,58$ & $2.550,56$ & $2.494,23$ & $2.331,93$ & $2.318,90$ & $2.228,38$ & $2.172,91$ & $2.143,83$ \\
\hline $53.958,20$ & $56.890,85$ & $56.005,79$ & $55.515,17$ & $54.684,78$ & $54.941,21$ & $54.505,50$ & $55.370,05$ & $55.643,79$ & $53.701,37$ \\
\hline 729,30 & 663,36 & 669,97 & 679,28 & 709,17 & 583,22 & 422,01 & 532,72 & 606,97 & 504,08 \\
\hline $1.026,90$ & 984,62 & 975,84 & $1.080,48$ & $1.119,48$ & $1.181,21$ & $1.303,14$ & $1.285,24$ & $1.267,43$ & $1.206,20$ \\
\hline $9.358,86$ & $9.219,21$ & $7.478,13$ & $7.226,49$ & $7.159,56$ & $7.245,93$ & $7.436,94$ & $7.411,51$ & $7.158,01$ & $6.824,14$ \\
\hline $5.914,50$ & $5.527,71$ & $5.375,27$ & $5.342,00$ & $5.427,46$ & $5.990,05$ & $6.105,47$ & $5.806,74$ & $5.552,15$ & $5.603,09$ \\
\hline $1.341,49$ & $1.288,55$ & $1.402,21$ & $1.305,18$ & $1.428,44$ & $1.277,63$ & $1.272,02$ & $1.345,20$ & $1.380,12$ & $1.200,45$ \\
\hline 423,41 & 373,29 & 410,71 & 430,98 & 447,85 & 418,18 & 562,89 & 545,01 & 514,80 & 504,04 \\
\hline $3.907,06$ & $4.525,86$ & $4.552,23$ & $4.384,76$ & $4.504,81$ & $4.427,91$ & $4.496,72$ & $4.534,04$ & $4.019,11$ & $3.469,73$ \\
\hline $5.917,37$ & $6.079,03$ & $6.077,26$ & $6.542,51$ & $6.007,40$ & $6.281,79$ & $6.396,59$ & $6.275,15$ & $5.719,30$ & $4.853,43$ \\
\hline $5.200,52$ & $5.442,53$ & $5.372,95$ & $5.641,89$ & $5.641,95$ & $5.341,58$ & 5.565 & 5.685 & $5.609,25$ & $5.410,83$ \\
\hline $13.464,80$ & $13.534,62$ & $14.013,66$ & $13.999,30$ & $14.800,05$ & $14.611,15$ & $14.044,62$ & $13.508,84$ & $13.384,47$ & $12.851,29$ \\
\hline $138.964,36$ & $147.030,52$ & $158.704,17$ & $175.299,48$ & $195.264,00$ & $181.210,48$ & $178.443,11$ & $176.635,22$ & $174.403,81$ & $164.226,65$ \\
\hline $9.819,77$ & $10.927,27$ & $11.115,08$ & $11.535,57$ & $11.542,55$ & $10.510,58$ & $10.531,80$ & $10.379,18$ & $10.363,98$ & $10.121,66$ \\
\hline $3.657,20$ & $3.792,84$ & $3.656,51$ & $3.679,30$ & $3.594,03$ & $3.267,15$ & $3.114,19$ & $3.132,67$ & $3.171,97$ & $3.366,19$ \\
\hline $78.484,25$ & $87.033,41$ & $91.060,09$ & $91.783,87$ & $88.341,50$ & $89.176,17$ & $90.046,03$ & $91.385,30$ & $93.408,16$ & $87.607,63$ \\
\hline 595.035 & 616.012 & 632.091 & 668.152 & 687.551 & 684.820 & 697.026 & 701.540 & 685.638 & 627.318 \\
\hline
\end{tabular}

Fonte: Elaboração própria dos autores a partir de dados do OECD.STAT. 